For Internal Distribution Only

Accelerator Division

Alternating Gradient Synchrotron Department BROOKHAVEN NATIONAL LABORATORY

Upton, New York 11973

Accelerator Division

Technical Note

AGS/AD/Tech. Note No. 429

THE QUADRUPOLE AND SEXTUPOLE FIELDS OF THE

AGS MAIN MAGNETS

R. Thern, E. Bleser

March 4, 1996 


\title{
THE QUADRUPOLE AND SEXTUPOLE FIELDS OF THE AGS MAIN MAGNETS
}

\author{
R. THERN, E. BLESER
}

March 4, 1996

Summary

This note records and parameterizes as a function of momentum the strength of the quadrupole and sextupole fields in the AGS main magnets.

The AGS Main Magnets

The AGS main magnets are combined function C-magnets which have two lengths. The "long" magnets are 90 inches long and are designated " $\mathrm{A}$ " or " $\mathrm{C}$ " magnets. The "short" magnets are 75 inches long and are designated " $\mathrm{B}$ " magnets. If the wide side of the magnet gap is to the outside of the magnet, away from the backleg, the magnet is called an open magnet. If the narrow side of the gap is to the outside of the magnet, the magnet is called a closed magnet. There are three kinds of magnets: long, open or closed, and short, open. Their designations are:
A long, open
B short, open
C long, closed.

Depending on their installation orientation in the ring, each of these magnets may be focusing or defocusing. Therefore there are six designations for magnets installed in the ring: $\mathrm{AD}, \mathrm{AF}, \mathrm{BD}$, $\mathrm{BF}, \mathrm{CD}$, and $\mathrm{CF}$.

The Measurement Data

Some years ago one of us (R.T.) made point-to-point field maps for an open magnet and a closed magnet over a number of current settings. The data were analyzed and used to generate the excitation functions and harmonic contents for the AGS main magnets. The results of this analysis have been incorporated into a number of operating systems, however the results have never been documented. Since magnetic storage may be long-lasting but the retrieval hardware and software seem to disappear in less than a decade, this note is being prepared to provide a permanent record of the data. It deals only with the analyzed results of quadrupole and sextupole component of the AGS main magnets. The dipole component is treated in AGS Tech. Note 424.

The Results

The results are given in the standard nomenclature, used for instance in MAD, of the quadrupole coefficient, $\mathrm{K} 1$, and the sextuple coefficient, $\mathrm{K} 2$, where: 


$$
K 1=\frac{1}{B \rho} \frac{d B}{d x}
$$

and

$$
K 2=\frac{1}{B \rho} \frac{d^{2} B}{d x^{2}}
$$

The results are found by point-to-point evaluation and integration along the arc of the reference orbit. The integration is performed along two different paths in each magnet: $\mathrm{F}$ for integration along the reference orbit when the magnet is focusing, and $\mathrm{D}$ for integration along the reference orbit when the magnet is defocusing. These paths are defined in Tech. Notes 215 and 217. Table 1 gives $\mathrm{K} 1$ for the six magnet configurations over a range of momenta. Table 2 gives $\mathrm{K} 2$, the sextupoles coefficient.

\section{Accuracy}

The point-to-point accuracy of the measurements is about one part in 5000. Integrating over the fields probably gives relative accuracies better than this. However the overall calibration of the apparatus is probably about $0.5 \%$. Fitting the coefficients as a function of momentum introduces additional uncertainties leading to the conclusion that the overall accuracy in predicting tunes should be better than one part in a thousand. Careful tune measurements may approach a few parts in ten thousand. Thus the tune data may be somewhat better than the predictions based on field measurements and we may want to adjust slightly the results presented in this note. In this note we carry a lot of decimal places for two reasons: we can avoid round-off problems in the parameterizations and many decimal places will enable us to identify the source of the data in the future.

\section{Parameterization}

For the quadrupole coefficient, K1, Figure 1 shows the data from Table 1 . We have parameterized these curves by fitting the low field data to powers of $1 / P$, forcing the curve to be a straight line from 6 to $15 \mathrm{GeV} / \mathrm{c}$, and fitting the remainder to a power series in $\mathrm{P}$. The fitted curves are also shown in Figure 1 and the parameters are given in Table 3.

$$
K 1=\frac{q_{-2}}{P^{2}}+\frac{q_{-1}}{P}+q_{0}+q_{1} P+q_{2} P^{2}+q_{3} P^{3}+q_{4} P^{4}+q_{5} P^{5}+q_{6} P^{6}
$$


For the sextuple coefficient, K2, Figure 2 shows the data from Table 2. We have parameterized these curves by fitting the low field data to powers of $1 / \mathrm{P}$ and fitting the remainder to a power series in $P$. The fitted curves are also shown in Figure 2 and the parameters are given in Table 4.

$$
K 2=\frac{s_{-3}}{P^{3}}+\frac{s_{-2}}{P^{2}}+\frac{s_{-1}}{P}+s_{0}+s_{1} P+s_{2} P^{2}+s_{3} P^{3}+s_{4} P^{4}+s_{5} P^{5}+s_{6} P^{6}
$$

We have chosen to use the momentum of the central orbit, $\mathrm{P}$ in $\mathrm{GeV} / \mathrm{c}$, as our standard variable. In this and subsequent notes machine properties will be recorded as a function of $P$. Tech Note 424 gives the formulas for relating the field, B, and the current, I, to P. 
TABLE 1

\section{QUADRUPOLE COEFFICIENTS of AGS MAIN MAGNETS}

\begin{tabular}{|r|c|c|c|c|c|c|c|}
\hline \multicolumn{1}{|c|}{$\mathbf{I}$} & $\mathbf{P}$ & $\mathbf{K 1 A D}$ & $\mathbf{K 1 A F}$ & $\mathbf{K 1 B D}$ & $\mathbf{K 1 B F}$ & $\mathbf{K 1 C D}$ & K1CF \\
\hline AMPS & $\mathbf{G e V / c}$ & $\mathbf{m}^{-\mathbf{2}}$ & $\mathbf{m}^{-2}$ & $\mathbf{m}^{-2}$ & $\mathbf{m}^{-\mathbf{2}}$ & $\mathbf{m}^{-\mathbf{2}}$ & $\mathbf{m}^{\mathbf{2}}$ \\
\hline 15 & 0.212 & $-4.7180 \mathrm{E}-02$ & $4.7274 \mathrm{E}-02$ & $-4.6954 \mathrm{E}-02$ & $4.7033 \mathrm{E}-02$ & $-5.0568 \mathrm{E}-02$ & $5.0680 \mathrm{E}-02$ \\
\hline 16 & 0.223 & $-4.7277 \mathrm{E}-02$ & $4.7368 \mathrm{E}-02$ & $-4.7056 \mathrm{E}-02$ & $4.7133 \mathrm{E}-02$ & $-5.0455 \mathrm{E}-02$ & $5.0562 \mathrm{E}-02$ \\
\hline 17 & 0.234 & $-4.7352 \mathrm{E}-02$ & $4.7441 \mathrm{E}-02$ & $-4.7137 \mathrm{E}-02$ & $4.7211 \mathrm{E}-02$ & $-5.0520 \mathrm{E}-02$ & $5.0634 \mathrm{E}-02$ \\
\hline 19 & 0.257 & $-4.7454 \mathrm{E}-02$ & $4.7540 \mathrm{E}-02$ & $-4.7234 \mathrm{E}-02$ & $4.7307 \mathrm{E}-02$ & $-5.0125 \mathrm{E}-02$ & $5.0229 \mathrm{E}-02$ \\
\hline 30 & 0.381 & $-4.7874 \mathrm{E}-02$ & $4.7947 \mathrm{E}-02$ & $-4.7664 \mathrm{E}-02$ & $4.7725 \mathrm{E}-02$ & $-4.9465 \mathrm{E}-02$ & $4.9557 \mathrm{E}-02$ \\
\hline 50 & 0.606 & $-4.8248 \mathrm{E}-02$ & $4.8312 \mathrm{E}-02$ & $-4.8050 \mathrm{E}-02$ & $4.8104 \mathrm{E}-02$ & $-4.9168 \mathrm{E}-02$ & $4.9244 \mathrm{E}-02$ \\
\hline 54 & 0.651 & $-4.8285 \mathrm{E}-02$ & $4.8348 \mathrm{E}-02$ & $-4.8087 \mathrm{E}-02$ & $4.8141 \mathrm{E}-02$ & $-4.9106 \mathrm{E}-02$ & $4.9181 \mathrm{E}-02$ \\
\hline 58 & 0.696 & $-4.8315 \mathrm{E}-02$ & $4.8378 \mathrm{E}-02$ & $-4.8124 \mathrm{E}-02$ & $4.8177 \mathrm{E}-02$ & $-4.9070 \mathrm{E}-02$ & $4.9142 \mathrm{E}-02$ \\
\hline 180 & 2.096 & $-4.8602 \mathrm{E}-02$ & $4.8658 \mathrm{E}-02$ & $-4.8415 \mathrm{E}-02$ & $4.8463 \mathrm{E}-02$ & $-4.8604 \mathrm{E}-02$ & $4.8673 \mathrm{E}-02$ \\
\hline 500 & 5.799 & $-4.8681 \mathrm{E}-02$ & $4.8733 \mathrm{E}-02$ & $-4.8495 \mathrm{E}-02$ & $4.8540 \mathrm{E}-02$ & $-4.8525 \mathrm{E}-02$ & $4.8594 \mathrm{E}-02$ \\
\hline 1325 & 15.360 & $-4.8692 \mathrm{E}-02$ & $4.8746 \mathrm{E}-02$ & $-4.8503 \mathrm{E}-02$ & $4.8550 \mathrm{E}-02$ & $-4.8491 \mathrm{E}-02$ & $4.8557 \mathrm{E}-02$ \\
\hline 1775 & 20.542 & $-4.8638 \mathrm{E}-02$ & $4.8697 \mathrm{E}-02$ & $-4.8442 \mathrm{E}-02$ & $4.8493 \mathrm{E}-02$ & $-4.8457 \mathrm{E}-02$ & $4.8529 \mathrm{E}-02$ \\
\hline 2225 & 25.615 & $-4.8490 \mathrm{E}-02$ & $4.8560 \mathrm{E}-02$ & $-4.8275 \mathrm{E}-02$ & $4.8335 \mathrm{E}-02$ & $-4.8332 \mathrm{E}-02$ & $4.8415 \mathrm{E}-02$ \\
\hline 2400 & 27.508 & $-4.8375 \mathrm{E}-02$ & $4.8450 \mathrm{E}-02$ & $-4.8146 \mathrm{E}-02$ & $4.8210 \mathrm{E}-02$ & $-4.8207 \mathrm{E}-02$ & $4.8295 \mathrm{E}-02$ \\
\hline 2575 & 29.262 & $-4.8170 \mathrm{E}-02$ & $4.8247 \mathrm{E}-02$ & $-4.7924 \mathrm{E}-02$ & $4.7989 \mathrm{E}-02$ & $-4.8018 \mathrm{E}-02$ & $4.8108 \mathrm{E}-02$ \\
\hline 2925 & 32.107 & $-4.7483 \mathrm{E}-02$ & $4.7557 \mathrm{E}-02$ & $-4.7217 \mathrm{E}-02$ & $4.7280 \mathrm{E}-02$ & $-4.7257 \mathrm{E}-02$ & $4.7341 \mathrm{E}-02$ \\
\hline
\end{tabular}

TABLE 2

SEXTUPOLE COEFFICIENTS of AGS MAGNETS

\begin{tabular}{|r|c|c|c|c|c|c|c|}
\hline \multicolumn{1}{|c|}{$\mathbf{I}$} & $\mathbf{P}$ & $\mathbf{K} 2 \mathrm{AD}$ & $\mathbf{K} 2 \mathrm{AF}$ & $\mathbf{K} 2 \mathrm{BD}$ & $\mathbf{K D B F}$ & $\mathbf{K} \mathbf{C D}$ & $\mathbf{K} 2 \mathrm{CF}$ \\
\hline AMPS & $\mathbf{G e V / c}$ & $\mathbf{m}-\mathbf{3}$ & $\mathbf{m}-\mathbf{3}$ & $\mathbf{m}-\mathbf{3}$ & $\mathbf{m}-\mathbf{3}$ & $\mathbf{m}-\mathbf{3}$ & $\mathbf{m}-\mathbf{3}$ \\
\hline 15 & 0.212 & $-2.671 \mathrm{E}-02$ & $-2.690 \mathrm{E}-02$ & $-2.828 \mathrm{E}-02$ & $-2.840 \mathrm{E}-02$ & $9.613 \mathrm{E}-03$ & $9.444 \mathrm{E}-03$ \\
\hline 16 & 0.223 & $-2.546 \mathrm{E}-02$ & $-2.598 \mathrm{E}-02$ & $-2.684 \mathrm{E}-02$ & $-2.724 \mathrm{E}-02$ & $8.980 \mathrm{E}-03$ & $8.504 \mathrm{E}-03$ \\
\hline 17 & 0.234 & $-2.503 \mathrm{E}-02$ & $-2.529 \mathrm{E}-02$ & $-2.653 \mathrm{E}-02$ & $-2.681 \mathrm{E}-02$ & $6.461 \mathrm{E}-03$ & $7.069 \mathrm{E}-03$ \\
\hline 19 & 0.257 & $-2.319 \mathrm{E}-02$ & $-2.364 \mathrm{E}-02$ & $-2.469 \mathrm{E}-02$ & $-2.505 \mathrm{E}-02$ & $6.143 \mathrm{E}-03$ & $5.929 \mathrm{E}-03$ \\
\hline 30 & 0.381 & $-1.778 \mathrm{E}-02$ & $-1.809 \mathrm{E}-02$ & $-1.931 \mathrm{E}-02$ & $-1.957 \mathrm{E}-02$ & $1.465 \mathrm{E}-05$ & $-2.093 \mathrm{E}-04$ \\
\hline 50 & 0.606 & $-1.372 \mathrm{E}-02$ & $-1.394 \mathrm{E}-02$ & $-1.522 \mathrm{E}-02$ & $-1.541 \mathrm{E}-02$ & $-3.276 \mathrm{E}-03$ & $-3.440 \mathrm{E}-03$ \\
\hline 54 & 0.651 & $-1.349 \mathrm{E}-02$ & $-1.368 \mathrm{E}-02$ & $-1.498 \mathrm{E}-02$ & $-1.517 \mathrm{E}-02$ & $-4.266 \mathrm{E}-03$ & $-4.423 \mathrm{E}-03$ \\
\hline 58 & 0.696 & $-1.328 \mathrm{E}-02$ & $-1.338 \mathrm{E}-02$ & $-1.477 \mathrm{E}-02$ & $-1.493 \mathrm{E}-02$ & $-4.440 \mathrm{E}-03$ & $-4.519 \mathrm{E}-03$ \\
\hline 180 & 2.096 & $-8.550 \mathrm{E}-03$ & $-8.676 \mathrm{E}-03$ & $-9.951 \mathrm{E}-03$ & $-1.006 \mathrm{E}-02$ & $-8.734 \mathrm{E}-03$ & $-8.845 \mathrm{E}-03$ \\
\hline 500 & 5.799 & $-7.164 \mathrm{E}-03$ & $-7.253 \mathrm{E}-03$ & $-8.514 \mathrm{E}-03$ & $-8.605 \mathrm{E}-03$ & $-9.531 \mathrm{E}-03$ & $-9.625 \mathrm{E}-03$ \\
\hline 1325 & 15.360 & $-7.153 \mathrm{E}-03$ & $-7.297 \mathrm{E}-03$ & $-8.572 \mathrm{E}-03$ & $-8.700 \mathrm{E}-03$ & $-9.392 \mathrm{E}-03$ & $-9.620 \mathrm{E}-03$ \\
\hline 1775 & 20.542 & $-8.399 \mathrm{E}-03$ & $-8.412 \mathrm{E}-03$ & $-9.944 \mathrm{E}-03$ & $-9.963 \mathrm{E}-03$ & $-1.031 \mathrm{E}-02$ & $-1.045 \mathrm{E}-02$ \\
\hline 2225 & 25.615 & $-1.148 \mathrm{E}-02$ & $-1.136 \mathrm{E}-02$ & $-1.342 \mathrm{E}-02$ & $-1.333 \mathrm{E}-02$ & $-1.319 \mathrm{E}-02$ & $-1.318 \mathrm{E}-02$ \\
\hline 2400 & 27.508 & $-1.451 \mathrm{E}-02$ & $-1.436 \mathrm{E}-02$ & $-1.667 \mathrm{E}-02$ & $-1.656 \mathrm{E}-02$ & $-1.633 \mathrm{E}-02$ & $-1.631 \mathrm{E}-02$ \\
\hline 2575 & 29.262 & $-1.991 \mathrm{E}-02$ & $-1.984 \mathrm{E}-02$ & $-2.227 \mathrm{E}-02$ & $-2.222 \mathrm{E}-02$ & $-2.227 \mathrm{E}-02$ & $-2.218 \mathrm{E}-02$ \\
\hline 2925 & 32.107 & $-3.405 \mathrm{E}-02$ & $-3.397 \mathrm{E}-02$ & $-3.656 \mathrm{E}-02$ & $-3.650 \mathrm{E}-02$ & $-3.676 \mathrm{E}-02$ & $-3.692 \mathrm{E}-02$ \\
\hline
\end{tabular}


TABLE 3

FITTED PARAMETERS for CALCULATING K1versus $\mathbf{P}$

\begin{tabular}{|c|c|r|r|r|r|r|}
\hline $\begin{array}{c}\text { PARA- } \\
\text { METER }\end{array}$ & \multicolumn{1}{c|}{ K1AD } & \multicolumn{1}{c|}{ K1AF } & \multicolumn{1}{c|}{ K1BD } & \multicolumn{1}{c|}{ K1BF } & \multicolumn{1}{c|}{ K1CD } & \multicolumn{1}{c|}{ K1CF } \\
\cline { 2 - 7 } & $\mathbf{m}^{-2}$ & $\mathbf{m}^{-2}$ & $\mathbf{m}^{-2}$ & $\mathbf{m}^{-2}$ & $\mathbf{m}^{-2}$ & $\mathbf{m}^{-2}$ \\
\hline $\mathrm{q}_{-2}$ & $1.04942 \mathrm{E}-05$ & $-9.96282 \mathrm{E}-06$ & $1.08550 \mathrm{E}-05$ & $-1.03703 \mathrm{E}-05$ & $-1.81597 \mathrm{E}-05$ & $1.93892 \mathrm{E}-05$ \\
\hline $\mathrm{q}_{-1}$ & $2.77647 \mathrm{E}-04$ & $-2.71244 \mathrm{E}-04$ & $2.83918 \mathrm{E}-04$ & $-2.79087 \mathrm{E}-04$ & $-3.68214 \mathrm{E}-04$ & $3.72630 \mathrm{E}-04$ \\
\hline $\mathrm{q}_{0}$ & $-4.87187 \mathrm{E}-02$ & $4.87709 \mathrm{E}-02$ & $-4.85320 \mathrm{E}-02$ & $4.85774 \mathrm{E}-02$ & $-4.84683 \mathrm{E}-02$ & $4.85356 \mathrm{E}-02$ \\
\hline $\mathrm{q}_{1}$ & $-4.42232 \mathrm{E}-05$ & $4.25198 \mathrm{E}-05$ & $-4.32894 \mathrm{E}-05$ & $4.18073 \mathrm{E}-05$ & $-1.48047 \mathrm{E}-05$ & $1.56503 \mathrm{E}-05$ \\
\hline $\mathrm{q}_{2}$ & $1.54321 \mathrm{E}-05$ & $-1.50273 \mathrm{E}-05$ & $1.49027 \mathrm{E}-05$ & $-1.45470 \mathrm{E}-05$ & $6.80258 \mathrm{E}-06$ & $-7.01344 \mathrm{E}-06$ \\
\hline $\mathrm{q}_{3}$ & $-2.02357 \mathrm{E}-06$ & $1.99004 \mathrm{E}-06$ & $-1.93514 \mathrm{E}-06$ & $1.90478 \mathrm{E}-06$ & $-1.10180 \mathrm{E}-06$ & $1.12750 \mathrm{E}-06$ \\
\hline $\mathrm{q}_{4}$ & $1.25812 \mathrm{E}-07$ & $-1.24749 \mathrm{E}-07$ & $1.19518 \mathrm{E}-07$ & $-1.18469 \mathrm{E}-07$ & $8.02484 \mathrm{E}-08$ & $-8.21732 \mathrm{E}-08$ \\
\hline $\mathrm{q}_{5}$ & $-3.72654 \mathrm{E}-09$ & $3.72397 \mathrm{E}-09$ & $-3.52356 \mathrm{E}-09$ & $3.51615 \mathrm{E}-09$ & $-2.69635 \mathrm{E}-09$ & $2.77133 \mathrm{E}-09$ \\
\hline $\mathrm{q}_{6}$ & $4.30540 \mathrm{E}-11$ & $-4.33033 \mathrm{E}-11$ & $4.07089 \mathrm{E}-11$ & $-4.08535 \mathrm{E}-11$ & $3.45700 \mathrm{E}-11$ & $-3.56256 \mathrm{E}-11$ \\
\hline
\end{tabular}

TABLE 4

FITTED PARAMETERS for CALCULATING K2 versus $P$

\begin{tabular}{|c|r|r|r|r|r|r|}
\hline PARA- & \multicolumn{1}{c|}{ K2AD } & \multicolumn{1}{c|}{ K2AF } & \multicolumn{1}{c|}{ K2BD } & \multicolumn{1}{c|}{ K2BF } & \multicolumn{1}{c|}{ K2CD } & \multicolumn{1}{c|}{ K2CF } \\
\cline { 2 - 6 } METER & \multicolumn{1}{c|}{$\mathbf{m}^{-3}$} & $\mathbf{m}^{-3}$ & $\mathbf{m}^{-3}$ & $\mathbf{m}^{-3}$ & \multicolumn{1}{c|}{$\mathbf{m}^{-3}$} & $\mathbf{m}^{-3}$ \\
\hline $\mathrm{s}_{-3}$ & $-4.541 \mathrm{E}-05$ & $-2.112 \mathrm{E}-05$ & $-5.333 \mathrm{E}-05$ & $-3.360 \mathrm{E}-05$ & $5.462 \mathrm{E}-05$ & $3.241 \mathrm{E}-05$ \\
\hline $\mathrm{s}_{-2}$ & $3.866 \mathrm{E}-04$ & $2.313 \mathrm{E}-04$ & $4.614 \mathrm{E}-04$ & $3.420 \mathrm{E}-04$ & $-3.118 \mathrm{E}-04$ & $-1.491 \mathrm{E}-04$ \\
\hline $\mathrm{s}_{-1}$ & $-5.152 \mathrm{E}-03$ & $-4.989 \mathrm{E}-03$ & $-5.368 \mathrm{E}-03$ & $-5.262 \mathrm{E}-03$ & $4.530 \mathrm{E}-03$ & $4.230 \mathrm{E}-03$ \\
\hline $\mathrm{s}_{0}$ & $-6.237 \mathrm{E}-03$ & $-6.356 \mathrm{E}-03$ & $-7.548 \mathrm{E}-03$ & $-7.659 \mathrm{E}-03$ & $-1.033 \mathrm{E}-02$ & $-1.036 \mathrm{E}-02$ \\
\hline $\mathrm{s}_{1}$ & $-8.211 \mathrm{E}-05$ & $-1.355 \mathrm{E}-04$ & $-8.463 \mathrm{E}-05$ & $-1.202 \mathrm{E}-04$ & $-5.104 \mathrm{E}-04$ & $-4.559 \mathrm{E}-04$ \\
\hline $\mathrm{s}_{2}$ & $2.948 \mathrm{E}-05$ & $5.202 \mathrm{E}-05$ & $3.258 \mathrm{E}-05$ & $4.837 \mathrm{E}-05$ & $1.748 \mathrm{E}-04$ & $1.559 \mathrm{E}-04$ \\
\hline $\mathrm{s}_{3}$ & $-2.636 \mathrm{E}-06$ & $-5.935 \mathrm{E}-06$ & $-3.462 \mathrm{E}-06$ & $-5.852 \mathrm{E}-06$ & $-1.907 \mathrm{E}-05$ & $-1.702 \mathrm{E}-05$ \\
\hline $\mathrm{s}_{4}$ & $2.178 \mathrm{E}-09$ & $2.124 \mathrm{E}-07$ & $7.609 \mathrm{E}-08$ & $2.318 \mathrm{E}-07$ & $9.025 \mathrm{E}-07$ & $7.920 \mathrm{E}-07$ \\
\hline $\mathrm{s}_{5}$ & $6.024 \mathrm{E}-09$ & $6.365 \mathrm{E}-11$ & $3.240 \mathrm{E}-09$ & $-1.237 \mathrm{E}-09$ & $-1.751 \mathrm{E}-08$ & $-1.445 \mathrm{E}-08$ \\
\hline $\mathrm{s}_{6}$ & $-1.607 \mathrm{E}-10$ & $-9.882 \mathrm{E}-11$ & $-1.247 \mathrm{E}-10$ & $-7.770 \mathrm{E}-11$ & $7.240 \mathrm{E}-11$ & $3.833 \mathrm{E}-11$ \\
\hline
\end{tabular}


FIGURE 1.

QUADRUPOLE COEFFICIENTS
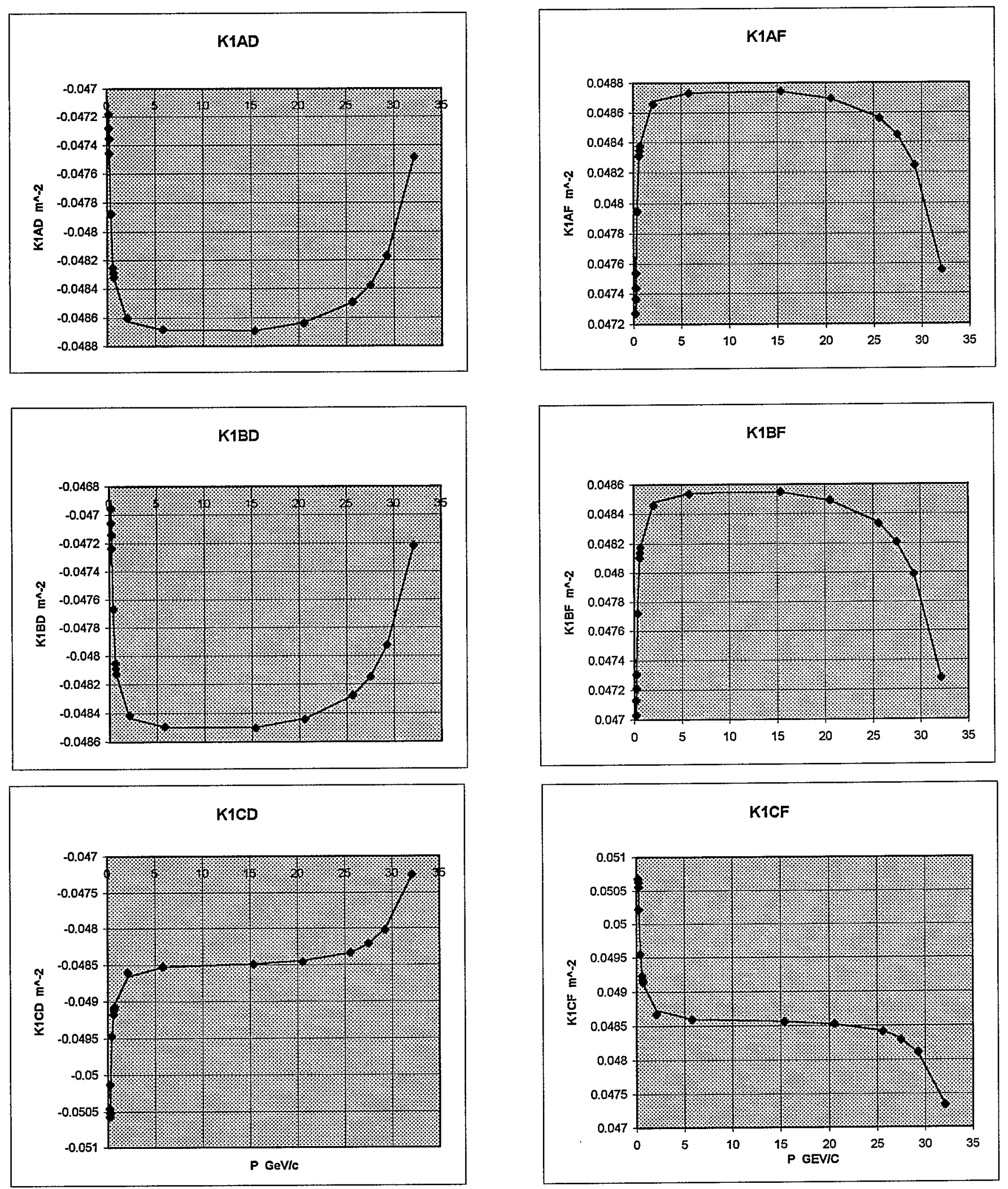

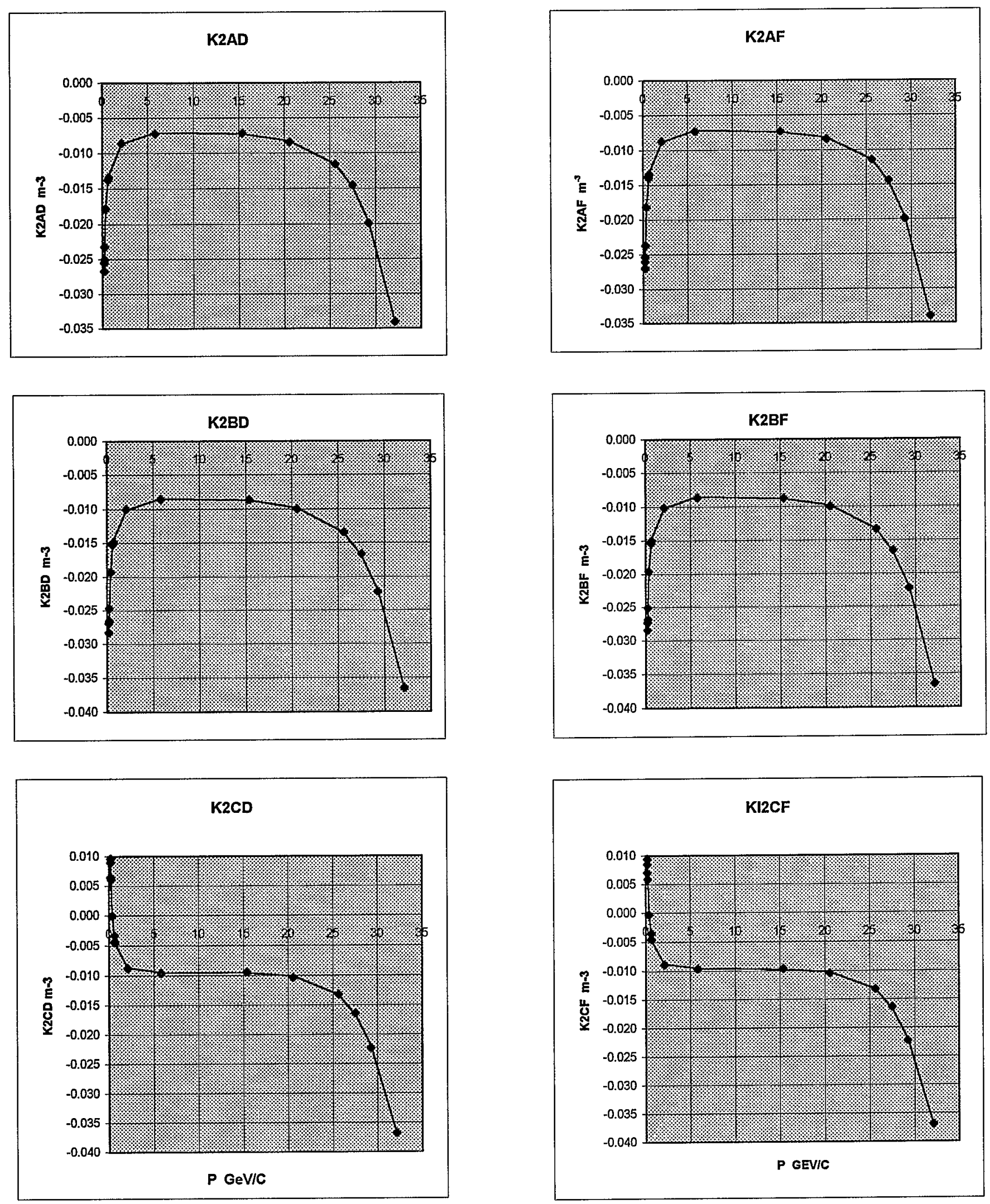